\title{
How to stay out of trouble in RIXS calculations within equation-of-motion coupled-cluster damped response theory? Safe hitchhiking in the excitation manifold by means of core-valence separation
}

\author{
Kaushik D. Nanda ${ }^{a, *}$, Marta L. Vidal ${ }^{b}$, Rasmus Faber ${ }^{b}$, Sonia Coriani ${ }^{b}$, and Anna I. Krylov ${ }^{a, c}$ \\ ${ }^{a}$ Department of Chemistry, University of Southern California, Los Angeles, California 90089-0482 \\ ${ }^{b}$ DTU Chemistry - Department of Chemistry, Technical \\ University of Denmark, DK-2800, Kongens Lyngby, Denmark \\ ${ }^{c}$ The Hamburg Centre for Ultrafast Imaging, Luruper Chaussee 149, 22671 Hamburg, Germany \\ * E-mail: kaushikdnanda@gmail.com (K.D.N.)
}

We present a novel approach for computing resonant inelastic X-ray scattering (RIXS) cross sections within the equation-of-motion coupled-cluster (EOM-CC) framework. The approach is based on recasting the sum-over-state expressions for RIXS moments (expressed via the EOM-CC resolvent) into a compact form by using damped response theory. Damped response formalism allows one to circumvent problems of divergent behavior of the response equation in the resonant regime. However, the convergence of response equations in the X-ray frequency range is often erratic due to the electronically metastable (i.e., resonant) nature of the virtual core-excited states embedded in the valence ionization continuum. We circumvent this problematic behavior by extending the core-valence separation (CVS) scheme, which decouples the valence-occupied and core-occupied excitation manifolds, into the response domain. The accuracy of the CVS-enabled damped response theory, implemented within the EOM-EE-CCSD (EOM-CC for excitation energies with single and double excitations) framework, is assessed by comparison against standard damped EOM-EE-CCSD response calculations. The capabilities of the new approach are illustrated by calculations of RIXS cross sections for benzene and benzene radical cation. 


\section{INTRODUCTION}

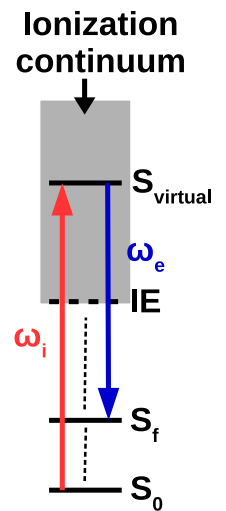

FIG. 1: RIXS is a non-linear process involving two photons. The excitation photon promotes the system from the ground state $S_{0}$ into the virtual state $S_{\text {virtual }}$ and the system emits a photon by relaxing into an excited state $\mathrm{S}_{f}$. The difference in the incident and emitted photon frequencies equals the excitation energy of final state (Eq. (1)). The system can also relax back to the ground state giving rise to the REXS (resonant elastic X-ray scattering) signal. Whereas the final excited states that give rise to the dominant spectral features lie below the ionization energy and are, therefore, bound with respect to electron ejection, the high-lying core-excited intermediate state $\mathrm{S}_{\text {virtual }}$ is embedded inside the ionization continuum shown by the gray box (valence resonances above the ionization onset can also contribute to the RIXS spectra).

Resonant inelastic X-ray scattering ${ }^{1-5}$ (RIXS) is a non-linear two-photon spectroscopy based on transitions involving core-level states. As illustrated in Fig. 1, RIXS is a onephoton-in/one-photon-out process. The energy difference between the absorbed and emitted photons ( $\omega_{i}$ and $\omega_{e}$, respectively) characterizes the differences between the valence electronic states:

$$
\omega_{i}-\omega_{e}=E_{f}-E_{0} .
$$

By virtue of using X-ray photons, RIXS exploits the highly localized and element-specific nature of the core orbitals ${ }^{6}$. Although RIXS is a coherent scattering process, it can be thought of as an initial excitation of a core-level electron into a valence orbital followed by radiative decay of another valence electron to fill the core hole. This molecular orbital picture

$95 \dagger$ See DOI: $10.1039 / \mathrm{cXCP00000x/}$ 


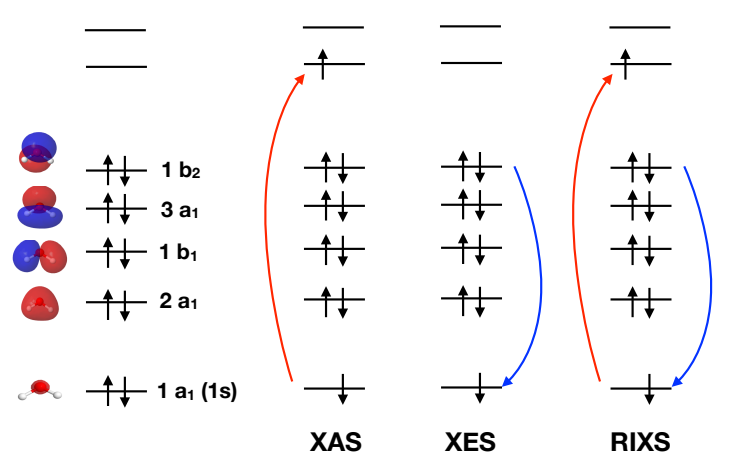

FIG. 2: Molecular orbital picture of the XAS, XES, and RIXS processes illustrated by using water. XAS entails excitation of core electrons into virtual molecular orbitals. The XES signal is produced by the relaxation of valence electrons into the core hole. RIXS process can be thought of as a two-step process coherently combining XAS and XES events.

is shown in Fig. 2 using water as an example: the rightmost panel shows the electronic configuration of the virtual state $\mathrm{S}_{\text {virtual }}$ and the red and blue arrows illustrate absorption and emission transitions. In this respect, one can (crudely) describe RIXS as simultaneous union of the X-ray absorption and X-ray emission events. This connection between XAS (Xray absorption spectroscopy), XES (X-ray emission spectroscopy), and RIXS is illustrated in Fig. 2. Thus, RIXS spectroscopy probes the unoccupied valence orbitals as in XAS, the occupied valence orbitals as in XES, and their correlation. Owing to its non-linear nature, the selection rules in RIXS differ from those in linear spectroscopies. For example, RIXS can provide the information about the transitions between valence excited states that are optically dark in one-photon spectroscopies. The spatial localization of core orbitals and their sensitivity to the chemical environment endows RIXS with the sensitivity to probe the local environment of an atom, its oxidation state, and the bonding pattern around it. Initial applications of RIXS were limited to studying charge-transfer and crystal-field transitions in metal oxides ${ }^{4,5}$, however, advances in X-ray light sources and instrumentation have afforded studying ultrafast nuclear dynamics with RIXS ${ }^{7}$.

The increasing popularity of X-ray spectroscopies ${ }^{8-11}$ such as RIXS, XAS, and XES have stimulated the development of quantum-chemical theory and electronic structure tools for modeling these spectra ${ }^{12,13}$ As in the case of VUV-based techniques ${ }^{14}$, theoretical modeling is required to unambiguously relate experimental measurements to molecular structures. 
However, as nicely described in a recent review ${ }^{12}$ of the theoretical modeling of X-ray spectroscopies, accurate description of core states is much more challenging than the description of valence states. Despite significant progress, the theoretical tools are lagging behind the experimental capabilities, creating a bottleneck for maximizing the scientific impact of multibillion advanced light sources facilities.

The challenges for electronic structure methods in modeling molecular processes in the X-ray regime include the prevalence of open-shell electronic structure patterns, the difficulty in computing high-lying core-excited states with standard solvers ${ }^{15-17}$, orbital relaxation effects due to strongly perturbed atomic core, the sensitivity of the signal to environmental effects, and the metastable character of the core-excited states embedded inside the valence ionization continuum ${ }^{18,19}$. Furthermore, computational modeling of multiphoton X-ray processes, such as RIXS, faces additional challenges. As in the case of other non-linear optical phenomena, the RIXS transition moments formally depend on all states of the system by virtue of the sum-over-states expressions that appear in the second and higher orders of perturbation theory. This has two important consequences. On the fundamental level, the sum over states means that the non-linear properties are more sensitive to the approximations to the many-body problem than the first-order properties - the latter depend only on the wave functions of the two states involved in a transition whereas the former are sensitive to the entire spectrum of a model Hamiltonian. On the practical level, the sum-over-state expressions need to be either truncated (by retaining several, presumably dominant, terms) or recast into a closed form using response theory. Given the resonant nature of RIXS, the former (approximate) approach may provide a qualitatively correct answer, which is often exploited in computational studies using multi-reference wave functions; see e.g., 2022. However, it is difficult to evaluate a priori the loss of accuracy due to the truncation. Therefore, the response theory approach is more desirable ${ }^{12,23,24}$. Unfortunately, the more rigorous response formulation leads to increased complexity of the equations and computer codes. Finally, one faces the non-convergence of response solutions due to the coupling of the response states (or virtual states) with the valence ionization continuum.

Analytic implementations of the state-of-the-art algebraic diagrammatic construction ${ }^{23}$ (ADC) and coupled-cluster ${ }^{24}$ methods for computing RIXS cross sections for small to medium-sized systems within the damped response theory ${ }^{25-28}$ framework have been reported. There is a continuing interest in less expensive approaches based on time-dependent 
density functional theory ${ }^{29,30}$ (TD-DFT) and DFT-parameterized wave-function methods ${ }^{31}$, however, the performance of TD-DFT methods in the X-ray domain is often negatively affected by the approximate treatment of the exchange-correlation ${ }^{32,33}$. Whereas numeric performance of analytic implementations based on damped response theory and correlated wave functions has been discussed and illustrated by examples ${ }^{23,25,26,28}$, the issue of diverging response solutions has remained hitherto unaddressed.

The equation-of-motion coupled-cluster (EOM-CC) methods ${ }^{34-39}$ provide a robust singlereference multi-state strategy for computing multiconfigurational wave functions, excitation energies, electron affinities, and ionization potentials of closed- and open-shell systems. Being a multi-state method, EOM-CC is an excellent platform for spectroscopy modeling because it describes multiple electronic states of different characters in a single computational framework; this leads to a balanced description of the states involved in transitions and also facilitates the calculations of the respective interstate properties. In contrast to multi-reference approaches, EOM-CC does not involve system-specific parameterization (e.g., active-space selection), thus satisfying Pople's requirements of theoretical model chemistry $^{40}$ that can be used for systematic studies and comparisons between different systems. The EOM-CC framework yields reliable lower-order properties such as solvatochromic shifts $^{41}$, transition dipole moments ${ }^{35}$, spin-orbit ${ }^{42-45}$ and non-adiabatic couplings ${ }^{46-48}$, as well as higher-order properties ${ }^{49}$ such as two-photon absorption cross sections ${ }^{50-55}$, static and dynamical polarizabilities ${ }^{56-59}$. Whereas the bulk of prior developments and applications of the EOM-CC methods as well as of the closely related coupled-cluster response theory ${ }^{60-62}$ were in the VUV regime, these methods are now being extended to the X-ray regime and their performance is being explored for computing, for example, $\mathrm{XAS}^{15-17,19,63-65}$, $\mathrm{XES}^{24}$, and RIXS $^{24}$ spectra.

Building on the (EOM-)CC damped response implementation reported by Faber and Coriani $^{24}$, here we present a novel damped response implementation for computing RIXS cross sections with a variant of the EOM-CC singles and doubles method for electronic excitation $^{35-37,66,67}$ (EOM-EE-CCSD) that provides converged response solutions by judicious exploitation of the excitation manifold. Our strategy to achieve robust convergence of the response equations in RIXS calculations is to employ the core-valence separation (CVS) scheme originally proposed by Cederbaum et al. ${ }^{68}$ This approach has been employed to compute resolved core-excited and core-ionized states within the framework of EOM-CC 
and CC-LR response theory ${ }^{19,64,69,70}$, but has not yet been employed for multiphoton X-ray processes such as RIXS. The CVS scheme projects out the valence ionization continuum and enables the resolution of core-excited (real or response) states. Specifically, we extend the recently reported CVS-EOM-EE-CCSD method with frozen-core (fc) approximation (fcCVS-EOM-EE-CCSD) $)^{19}$ to the calculation of RIXS cross sections. This idea is also being explored within the coupled-cluster singles and doubles complex-polarization propagator approach by Faber and Coriani ${ }^{71}$.

Here, we present the theory of RIXS cross sections within the EOM-EE-CCSD and fcCVS-EOM-EE-CCSD methods. We validate the implementation on a small test system $(\mathrm{LiH})$ and then compare the results of EOM-EE-CCSD and fc-CVS-EOM-EE-CCSD RIXS spectra for the water molecule. The method is implemented in the Q-Chem electronic structure package $\mathrm{e}^{72,73}$. Our production-level implementation is capable of treating both closed- and open-shell references. As an illustrative example, we compute and discuss RIXS spectra of benzene and its cation, highlighting the capabilities of the fc-CVS-EOM-EE-CCSD method. In the present study, we focus exclusively on the electronic factors entering RIXS cross sections; the extension of the theory to include vibronic effects, which are important for quantitative modeling of the spectra, will be carried out in future work.

\section{THEORY}

\section{A. CCSD and EOM-EE-CCSD theory}

The ground-state coupled-cluster wave function is given by

$$
\Psi_{0}=e^{T}\left|\Phi_{0}\right\rangle
$$

where $T$ is the cluster operator given in terms of creation $\left(a^{\dagger}, b^{\dagger}\right)$ and annihilation $(i, j)$ operators as follows:

$$
T=T_{1}+T_{2} ; \quad T_{1}=\sum_{i a} t_{i}^{a} a^{\dagger} i ; \quad T_{2}=\frac{1}{4} \sum_{i j a b} t_{i j}^{a b} a^{\dagger} b^{\dagger} j i
$$

The $T$ operator satisfies the following coupled-cluster equations:

$$
\left\langle\Phi_{\nu}|\bar{H}| \Phi_{0}\right\rangle=0
$$


where $\nu$ spans the singles and doubles excitation manifold for CCSD and $\bar{H}=e^{-T} H e^{T}$ is the similarity-transformed Hamiltonian. As usual, labels $a, b$ refer to virtual and $i, j$ to occupied moleculer (spin-)orbitals.

In the EOM-CCSD approach for excitation energies, the target excited states are described as excitations from the CCSD wave function as follows:

$$
L^{k} \bar{H}=L^{k} E_{k}
$$

and

$$
\bar{H} R^{k}=E_{k} R^{k}
$$

where $L$ and $R$ are the EOM-CCSD left (de-excitation), and EOM-CCSD right (excitation) operators

$$
L=l_{0}+L_{1}+L_{2} ; \quad L_{1}=\sum_{i a} l_{i}^{a} i^{\dagger} a ; \quad L_{2}=\frac{1}{4} \sum_{i j a b} l_{i j}^{a b} i^{\dagger} j^{\dagger} b a
$$

and

$$
\hat{R}=r_{0}+R_{1}+R_{2} ; \quad R_{1}=\sum_{i a} r_{i}^{a} a^{\dagger} i ; \quad R_{2}=\frac{1}{4} \sum_{i j a b} r_{i j}^{a b} a^{\dagger} b^{\dagger} j i,
$$

For the CCSD reference state $(k=0), R=r_{0}$ and $L=1+\Lambda$, where $\Lambda=\Lambda_{1}+\Lambda_{2}$ is the $\operatorname{CCSD} \Lambda$ operator. For EOM-CCSD states, $l_{0}=0$.

\section{B. RIXS within EOM-EE-CCSD damped response theory}

The RIXS (and REXS) cross section as a function of scattering angle $\theta$ (the angle between the polarization vector of the incoming photon and the propagation vector of the outgoing photon) is given in terms of RIXS transition strengths $S_{a b, c d}^{i f}$ as follows:

$$
\begin{aligned}
\sigma^{R I X S}(\theta)= & \frac{1}{15} \frac{\omega_{e}}{\omega_{i}} \sum_{x y}\left[\left(2-\frac{1}{2} \sin ^{2} \theta\right) S_{x y, x y}^{i f}\right. \\
& \left.+\left(\frac{3}{4} \sin ^{2} \theta-\frac{1}{2}\right)\left(S_{x y, y x}^{i f}+S_{x x, y y}^{i f}\right)\right],
\end{aligned}
$$

where $\omega_{e}$ and $\omega_{i}$ are frequencies of emitted and incident photons and the indices $x, y$ denote the Cartesian components ${ }^{23,24}$. If $\theta$ is defined as the angle between the polarization vector of the outgoing photon and the propagation vector of the incoming photon, $\cos ^{2} \theta$ should replace $0.5 \sin ^{2} \theta$ in the above expression ${ }^{74}$. 
Within the non-Hermitian EOM-CC theory, $S_{a b, c d}^{i f}$ are given by

$$
S_{a b, c d}^{i f}=\frac{1}{2}\left(M_{a b}^{i \leftarrow f} M_{c d}^{f \leftarrow i}+\left(M_{c d}^{i \leftarrow f}\right)^{*}\left(M_{a b}^{f \leftarrow i}\right)^{*}\right)
$$

where $^{*}$ denotes complex conjugation ${ }^{75,76}$. Here, $M_{a b}^{f \leftarrow i}$ and $M_{a b}^{i \leftarrow f}$ are the right and left RIXS moments given by the sum-over-state expressions, which within the EOM-CC framework assume the following form:

$$
\begin{aligned}
& M_{x y}^{f \leftarrow i}\left(\omega_{i, x}+i \gamma,-\omega_{e, y}-i \gamma\right) \\
& =-\sum_{n \geq 0}\left(\frac{\left\langle\Phi_{0} L^{f} e^{-T}\left|\mu^{y}\right| e^{T} R^{n} \Phi_{0}\right\rangle\left\langle\Phi_{0} L^{n} e^{-T}\left|\mu^{x}\right| e^{T} R^{i} \Phi_{0}\right\rangle}{\Omega_{n i}-\omega_{i, x}-i \gamma}\right. \\
& \left.\quad+\frac{\left\langle\Phi_{0} L^{f} e^{-T}\left|\mu^{x}\right| e^{T} R^{n} \Phi_{0}\right\rangle\left\langle\Phi_{0} L^{n} e^{-T}\left|\mu^{y}\right| e^{T} R^{i} \Phi_{0}\right\rangle}{\Omega_{n i}+\omega_{e, y}+i \gamma}\right)
\end{aligned}
$$

and

$$
\begin{aligned}
M_{x y}^{i \leftarrow f}\left(-\omega_{i, x}+i \gamma, \omega_{e, y}-i \gamma\right) & \\
=-\sum_{n \geq 0} & \left(\frac{\left\langle\Phi_{0} L^{i} e^{-T}\left|\mu_{x}\right| e^{T} R^{n} \Phi_{0}\right\rangle\left\langle\Phi_{0} L^{n} e^{-T}\left|\mu_{y}\right| e^{T} R^{f} \Phi_{0}\right\rangle}{\Omega_{n i}-\omega_{i, x}+i \gamma}\right. \\
& \left.+\frac{\left\langle\Phi_{0} L^{i} e^{-T}\left|\mu_{y}\right| e^{T} R^{n} \Phi_{0}\right\rangle\left\langle\Phi_{0} L^{n} e^{-T}\left|\mu_{x}\right| e^{T} R^{f} \Phi_{0}\right\rangle}{\Omega_{n i}+\omega_{e, y}-i \gamma}\right) .
\end{aligned}
$$

Here, $\Omega_{n m}=E_{n}-E_{m}$ is the energy difference between state $n$ and $m$ and the photon frequencies $\left(\omega\right.$ 's) are augmented with the phenomenological damping term ${ }^{77} i \gamma$.

Introducing the identity operator, $\hat{\mathbf{1}}=\sum_{\rho}\left|\Phi_{\rho}\right\rangle\left\langle\Phi_{\rho}\right|$, in Eqs. (10) and (11), we obtain

$$
\begin{aligned}
& M_{x y}^{f \leftarrow i}\left(\omega_{i, x}+i \gamma,-\omega_{e, y}-i \gamma\right)=-\sum_{\rho \nu} \\
& \quad\left\langle\Phi_{0} L^{f}\left|\bar{\mu}^{y}\right| \Phi_{\rho}\right\rangle \sum_{n \geq 0} \frac{\left\langle\Phi_{\rho} \mid R^{n} \Phi_{0}\right\rangle\left\langle\Phi_{0} L^{n} \mid \Phi_{\nu}\right\rangle}{\Omega_{n i}-\omega_{i, x}-i \gamma}\left\langle\Phi_{\nu}\left|\bar{\mu}^{x}\right| R^{i} \Phi_{0}\right\rangle+ \\
& \left.\quad\left\langle\Phi_{0} L^{f}\left|\bar{\mu}^{y}\right| \Phi_{\rho}\right\rangle \sum_{n \geq 0} \frac{\left\langle\Phi_{\rho} \mid R^{n} \Phi_{0}\right\rangle\left\langle\Phi_{0} L^{n} \mid \Phi_{\nu}\right\rangle}{\Omega_{n i}+\omega_{e, y}+i \gamma}\left\langle\Phi_{\nu}\left|\bar{\mu}^{x}\right| R^{i} \Phi_{0}\right\rangle\right)
\end{aligned}
$$

and

$$
\begin{aligned}
& M_{x y}^{i \leftarrow f}\left(-\omega_{i, x}+i \gamma, \omega_{e, y}-i \gamma\right)=-\sum_{\rho \nu} \\
& \quad\left(\left\langle\Phi_{0} L^{i}\left|\bar{\mu}^{x}\right| \Phi_{\rho}\right\rangle \sum_{n \geq 0} \frac{\left\langle\Phi_{\rho} \mid R^{n} \Phi_{0}\right\rangle\left\langle\Phi_{0} L^{n} \mid \Phi_{\nu}\right\rangle}{\Omega_{n i}-\omega_{i, x}+i \gamma}\left\langle\Phi_{\nu}\left|\bar{\mu}^{x}\right| R^{f} \Phi_{0}\right\rangle+\right. \\
& \left.\quad\left\langle\Phi_{0} L^{i}\left|\bar{\mu}^{x}\right| \Phi_{\rho}\right\rangle \sum_{n \geq 0} \frac{\left\langle\Phi_{\rho} \mid R^{n} \Phi_{0}\right\rangle\left\langle\Phi_{0} L^{n} \mid \Phi_{\nu}\right\rangle}{\Omega_{n i}+\omega_{e, y}-i \gamma}\left\langle\Phi_{\nu}\left|\bar{\mu}^{x}\right| R^{f} \Phi_{0}\right\rangle\right) .
\end{aligned}
$$


Here, $\bar{\mu}=e^{-T} \mu e^{T}$ is the similarity-transformed dipole-moment operator and $\rho$ spans the full excitation manifold, however, only the reference, singly, and doubly excited determinants survive for EOM-CCSD. Using the response intermediates defined $\mathrm{as}^{50}$

$$
\left[D^{x}\right]_{\nu}^{n}=\left\langle\Phi_{\nu}\left|\bar{\mu}^{x}\right| R^{n} \Phi_{0}\right\rangle
$$

and

$$
\left[\tilde{D}^{x}\right]_{\rho}^{n}=\left\langle\Phi_{0} L^{n}\left|\bar{\mu}^{x}\right| \Phi_{\rho}\right\rangle
$$

along with solutions, $X$ and $\tilde{X}$, of response equations given by

$$
\sum_{\nu}\left(\bar{H}-E_{0}-(\omega+i \gamma)\right)_{\rho \nu}\left(X_{r e}+i X_{i m}\right)_{\nu}=D_{\rho}
$$

and

$$
\sum_{\rho}\left(\tilde{X}_{r e}+i \tilde{X}_{i m}\right)_{\rho}\left(\bar{H}-E_{0}-(\omega+i \gamma)\right)_{\rho \nu}=\tilde{D}_{\nu}
$$

Eqs. (12) and (13) become

$$
\begin{aligned}
& M_{x y}^{f \leftarrow i}\left(\omega_{i, x}+i \gamma,-\omega_{e, y}-i \gamma\right) \\
& \quad=-\sum_{\rho}\left(\left[\tilde{D}^{y}\right]_{\rho}^{f}\left[X^{x}\left(\omega_{i, x}+i \gamma\right)\right]_{\rho}^{i}+\left[\tilde{D}^{x}\right]_{\rho}^{f}\left[X^{y}\left(-\omega_{e, y}-i \gamma\right)\right]_{\rho}^{i}\right) \\
& \quad=-\sum_{\rho}\left(\left[\tilde{X}^{y}\left(-\omega_{e, y}-i \gamma\right)\right]_{\rho}^{f}\left[D^{x}\right]_{\rho}^{i}+\left[\tilde{X}^{x}\left(\omega_{i, x}+i \gamma\right)\right]_{\rho}^{f}\left[D^{y}\right]_{\rho}^{i}\right)
\end{aligned}
$$

and

$$
\begin{aligned}
& M_{x y}^{i \leftarrow f}\left(-\omega_{i, x}+i \gamma, \omega_{e, y}-i \gamma\right) \\
& \quad=-\sum_{\rho}\left(\left[\tilde{D}^{x}\right]_{\rho}^{i}\left[X^{y}\left(\omega_{e, y}-i \gamma\right)\right]_{\rho}^{f}+\left[\tilde{D}^{y}\right]_{\rho}^{i}\left[X^{x}\left(-\omega_{i, x}+i \gamma\right)\right]_{\rho}^{f}\right) \\
& \quad=-\sum_{\rho}\left(\left[\tilde{X}^{x}\left(-\omega_{i, x}+i \gamma\right)\right]_{\rho}^{i}\left[D^{y}\right]_{\rho}^{f}+\left[\tilde{X}^{y}\left(\omega_{e, y}-i \gamma\right)\right]_{\rho}^{i}\left[D^{x}\right]_{\rho}^{f}\right),
\end{aligned}
$$

where $X=X_{\Re}+i X_{\Im}$ and $\tilde{X}=\tilde{X}_{\Re}+i \tilde{X}_{\Im}$. We solve the damped response equations (16) and (17) by modifying our standard DIIS procedure ${ }^{78,79}$ as described in the SI.

We note that the above damped response equations implicitly employ the following resolvent equation:

$$
\sum_{n \geq 0} \frac{\left\langle\Phi_{\rho} \mid R^{n} \Phi_{0}\right\rangle\left\langle\Phi_{0} L^{n} \mid \Phi_{\nu}\right\rangle}{\Omega_{n i}-\omega-i \gamma}=\left\langle\Phi_{\rho}\left|\left(\bar{H}-E_{0}-\omega-i \gamma\right)^{-1}\right| \Phi_{\nu}\right\rangle,
$$


which parallels our formal derivation of the $2 \mathrm{PA}$ response equations ${ }^{50}$. Here, the sum runs over all states of the system.

As a two-photon process, RIXS involves a virtual state (Fig. 1). Owing to the resonant nature of RIXS, the corresponding virtual state is a real core-excited high-lying state, which is a metastable state (specifically, Feshbach resonance) with respect to electron ejection embedded in the valence ionization continuum. This presence of the continuum cripples the convergence of the response solutions, $X$ and $\tilde{X}$, in Eqs. (16) and (17). Specifically, the problem manifests itself by oscillatory behavior of doubly excited amplitudes and by their large magnitude. We note that this problem does not arise in low-level theories without explicit double excitations ${ }^{18,71}$, such as CIS/TDDFT, ADC(2), or CC2 because the decay channels of core-level states involve two-electron transition. The damped response theory framework only mitigates the convergence issues due to resonant photons (i.e., singularities in the resolvent) but does not address the impact of the coupling with the continuum. To circumvent this problem, we employ CVS scheme within the response manifold.

\section{RIXS within fc-CVS-EOM-EE-CCSD damped response theory}

Within the fc-CVS-EOM-CCSD framework, we first impose the frozen-core (fc) approximation and create distinct core occupied $(C)$, valence occupied $(O)$, and unoccupied $(V)$ subspaces. As reported in Ref. 19, the reference, $C V, C O V V$, and $C C V V$ excitation manifolds are then used for computing the fc-CVS-EOM-CCSD states (below we refer to this configurational space as the CVS manifold). Note that in the fc-CVS-EOM-CCSD scheme, the $O V$ and $O O V V$ subspaces and, consequently, the valence continuum have been projected out, which blocks the decay channels and makes the fc-CVS-EOM-CCSD states bound. This separation of the bound and continuum configurations within CVS is akin to diabatization. It is also related to the Feshbach-Fano treatment of resonances ${ }^{80,81}$.

In the context of calculating RIXS moments, we employ the CVS scheme to approximate the sum-over-state expressions in Eqs. (10) and (11) such that only the fc-CVS-EOM-EECCSD states are included in the sum. The fc-CVS-EOM-CCSD resolvent equation is given 
by

$$
\begin{array}{r}
\frac{\left\langle\Phi_{\rho} \mid \Phi_{0}\right\rangle\left\langle\Phi_{0} \mid \Phi_{\nu}\right\rangle}{-\omega-i \gamma}+\sum_{n}^{C V S} \frac{\left\langle\Phi_{\rho} \mid R^{n} \Phi_{0}\right\rangle\left\langle\Phi_{0} L^{n} \mid \Phi_{\nu}\right\rangle}{\Omega_{n i}-\omega-i \gamma}= \\
\left\langle\Phi_{\rho}\left|\left(\bar{H}^{\mathrm{cvs}}-E_{0}-\omega-i \gamma\right)^{-1}\right| \Phi_{\nu}\right\rangle
\end{array}
$$

where the $\bar{H}^{\text {cvs }}$ is written in the basis of the reference and the CVS excitation manifold ( $C V$, $C O V V$, and $C C V V$ configurations). Eq. (21) reduces to

$$
\begin{array}{r}
\sum_{n}^{C V S} \frac{\left\langle\Phi_{\rho} \mid R^{n} \Phi_{0}\right\rangle\left\langle\Phi_{0} L^{n} \mid \Phi_{\nu}\right\rangle}{\Omega_{n i}-\omega-i \gamma}= \\
\left\langle\Phi_{\rho}\left|\left(\bar{H}^{\mathrm{cvs}}-E_{0}-\omega-i \gamma\right)^{-1}\right| \Phi_{\nu}\right\rangle-\frac{\delta_{0 \rho} \delta_{0 \nu}}{-\omega-i \gamma}
\end{array}
$$

The fc-CVS-EOM-EE-CCSD response equations are thus given by

$$
\sum_{\nu}\left(\bar{H}^{\mathrm{cvs}}-E_{0}-(\omega+i \gamma)\right)_{\rho \nu}\left(X_{\Re}+i X_{\Im}\right)_{\nu}=\left(1-\left|\Phi_{0}\right\rangle\left\langle\Phi_{0}\right|\right)_{\rho \lambda} D_{\lambda}
$$

and

$$
\sum_{\rho}\left(\tilde{X}_{\Re}+i \tilde{X}_{\Im}\right)_{\rho}\left(\bar{H}^{\mathrm{cvs}}-E_{0}-(\omega+i \gamma)\right)_{\rho \nu}=\tilde{D}_{\lambda}\left(1-\left|\Phi_{0}\right\rangle\left\langle\Phi_{0}\right|\right)_{\lambda \nu}
$$

where $\rho, \lambda$, and $\nu$ span the reference, $C V, C O V V$, and $C C V V$ excitation manifolds.

\section{COMPUTATIONAL DETAILS}

The validation calculations for $\mathrm{LiH}$ were carried out using the STO-3G basis and $r_{L i H}=1.6$ $\AA$. Water calculations were carried out using the same setup as in Ref. 24. Specifically, we computed RIXS cross sections including the lowest 20 excited states, using the geometry $\left(r_{O H}=0.95421 \AA, \angle_{H O H}=104.78^{\circ}\right)$ and basis set from Ref. 24 (also given in the SI). We use the EOM-EE-CCSD results reported at the incident photon frequency of $535.74 \mathrm{eV}$ that is resonant with the $1 s \rightarrow 4 a_{1}$ core excitation in water. The corresponding fc-CVS-EOMEE-CCSD incident photon frequency used in our calculations is $535.23 \mathrm{eV}$.

The calculations for neutral benzene were carried out at the geometry optimized with RI-MP2/cc-pVTZ. The calculations for the cation were carried out at two different geometries: one of the neutral (referred to as Franck-Condon (FC) structure) and one of the cation (optimized with EOM-IP-CCSD/cc-pVTZ). We considered the optimized geometry of the 
${ }^{2} \mathrm{~B}_{3 g}$ state, which is the lowest Jahn-Teller state (this structure can be described as "elongated", in terms of Ref. 82 or "acute $D_{2 h}$ ", in terms of Ref. 83). The optimized structure of the second Jahn-Teller state, ${ }^{2} \mathrm{~B}_{2 g}$, is nearly degenerate with the ${ }^{2} \mathrm{~B}_{3 g}$ structure and the barrier separating the two minima is well below zero-point energy ${ }^{83}$. Consequently, benzene cation's structure can be described by free pseudo-rotation ${ }^{83}$. We used the $6-311(2+,+) \mathrm{G}^{* *}$ basis with the uncontracted carbon core (denoted as 6-311(2+,+) $\mathrm{G}^{* *}(\mathrm{uC})$ ).

Depending on molecular orientation, symmetry labels corresponding to the same orbital or vibrational mode may be different. Q-Chem's standard molecular orientation is different from that of Mulliken ${ }^{84}$. For example, Q-Chem places water molecule in the $x z$ plane instead of the $y z$. Consequently, for $\mathrm{C}_{2 v}$ symmetry, $b_{1}$ and $b_{2}$ labels are flipped. Q-Chem places benzene in the $x y$ plane (with the $\mathrm{x}$-axis passing through the carbon atoms, see SI)) instead of the $y z$ plane. Consequently, the $b_{1 u}$ and $b_{3 u}$ labels are flipped in Q-Chem relative to the Mulliken convention. More details can be found at http://iopenshell.usc.edu/resources/howto/symmetry/. To avoid confusion with different molecular orientations and relabeling the states, here we report the structures and symmetry labels following the Q-Chem's notations.

The calculations of XAS were carried out using fc-CVS-EOM-EE-CCSD. For the cation, we tested restricted and unrestricted open-shell Hartree-Fock (ROHF and UHF, respectively) references and the difference between the two calculations was found to be small. Below we report the ROHF results. The XES transitions between different core and valence states were computed as the transitions between valence- and core-ionized states of the neutral reference, as suggested in Ref. 24. The valence states were described by EOM-IP-CCSD with frozen core and the core states were described by fc-CVS-EOM-IP-CCSD. The orbital character of the XAS and XES transitions was analyzed using Natural Transition Orbitals computed with the libwfa module ${ }^{85}$ and Dyson orbitals ${ }^{86,87}$.

The RIXS spectra were computed with UHF reference using the new fc-CVS-EOM-EECCSD scheme. All RIXS calculations for benzene were performed with $\gamma=0.01$ a.u. $=$ $0.272 \mathrm{eV}$. The cation's RIXS calculations were performed with $\gamma=0.005$ a.u. $=0.136 \mathrm{eV}$.

The stick spectra were convoluted with normalized Gaussian functions:

$$
\begin{aligned}
g(x) & =\frac{1}{\sigma \sqrt{2 \pi}} \exp \left(-\frac{\left(x-x_{0}\right)^{2}}{2 \sigma^{2}}\right) \\
F W H M & =2 \sqrt{2 \ln (2))} \sigma \approx 2.35482 \sigma
\end{aligned}
$$




\section{RESULTS AND DISCUSSION}

\section{A. Validation and benchmarks}

The calculation of RIXS cross sections using damped response theory combined with CVS is implemented in the Q-Chem electronic structure package ${ }^{72,73}$. Our productionlevel implementation builds upon the fc-CVS-EOM-CCSD suite of $\operatorname{codes}^{19}$ and the nonlinear response module ${ }^{50}$. The programmable equations are implemented using the general library for tensor computations, libtensor ${ }^{88}$. The implementation enables calculation of RIXS cross sections for both closed- and open-shell references. We validated our analytic damped response-theory approach against numeric sum-over-state calculations for the first excited state of LiH using the STO-3G basis; the results are given in the SI. The standard (i.e., full-valence) EOM-EE-CCSD implementation was also validated against Ref. 24.

To quantify the effect of using CVS within the response equations on the RIXS spectra, we compare the fc-CVS-EOM-EE-CCSD and standard EOM-EE-CCSD results for the water molecule. The incident photon frequency in each calculation is resonant with the $1 s_{O} \rightarrow 4 a_{1}$ core excitation. For this incident photon, the dominant RIXS transitions correspond to final states $1 \mathrm{~B}_{2}, 2 \mathrm{~A}_{1}$, and $3 \mathrm{~A}_{1}$ with orbital characters of $1 b_{2} \rightarrow 4 a_{1}, 3 a_{1} \rightarrow 4 a_{1}$, and $1 b_{2} \rightarrow 2 b_{2}$ (in Q-Chem's symmetry notation), respectively, as presented in Table I. Table I reveals that using the fc-CVS scheme has a minor impact on the RIXS cross sections for these dominant transitions in the spectrum. Although the effect on the cross sections of several minor bands is larger, the appearance of the overall computed spectrum is not affected by invoking CVS, as illustrated in Fig. 3 and by the two-dimensional RIXS map in the SI, which looks very similar to the one reported in Ref. 24. The changes in relative intensities of the major peaks due to different scattering angles $(\theta \mathrm{s})$ are also captured correctly.

Here we limit the comparison of the computed RIXS spectra to the full valence EOMCCSD damped response calculations ${ }^{24}$, because, although water is a popular benchmark molecule for theory ${ }^{23,24}$, it is not well suited for making comparisons with the experiments. The complications arise due to an anomalously strong effect of nuclear motions on its RIXS spectra $^{22}$. Similarly to valence ionization ${ }^{89}$, core-level excitation of water leads to ultrafast dissociation ${ }^{22}$. Because stretching of the $\mathrm{OH}$ bond strongly affects valence electronic states $^{22,33}$, this ultrafast motion has strong effect on the RIXS and XES lineshapes. Conse- 
TABLE I: Comparison of EOM-EE-CCSD and fc-CVS-EOM-EE-CCSD excitation energies and RIXS cross sections for $\mathrm{H}_{2} \mathrm{O}(\gamma=0.0045563$ a.u.). Incident photon frequency for EOM-EE-CCSD calculations $=19.6881298$ a.u. $(535.74 \mathrm{eV})$. Incident photon frequency for fc-CVS-EOM-EE-CCSD calculations $=19.6692301 \mathrm{a} . \mathrm{u}(535.23 \mathrm{eV})$. The columns marked 'ratio' give the ratio of computed RIXS cross sections with and without CVS.

\begin{tabular}{|c|c|c|c|c|c|c|c|c|c|c|c|}
\hline & \multicolumn{4}{|c|}{ EOM-EE-CCSD } & \multicolumn{7}{|c|}{ fc-CVS-EOM-EE-CCSD } \\
\hline Ex. & Ex.E. & $\sigma_{0^{\circ}}^{R I X S}$ & $\sigma_{45^{\circ}}^{R I X S}$ & $\sigma_{90^{\circ}}^{R I X S}$ & Ex. E. & $\sigma_{0^{\circ}}^{R I X S}$ & ratio & $\sigma_{45^{\circ}}^{R I X S}$ & ratio & $\sigma_{90^{\circ}}^{R I X S}$ & ratio \\
\hline St. ${ }^{a}$ & $\mathrm{eV}$ & a.u. & a.u. & a.u. & $\mathrm{eV}$ & a.u. & & a.u. & & a.u. & \\
\hline $1 \mathrm{~B}_{2}$ & 7.41 & 0.023556 & 0.020601 & 0.017646 & 7.40 & 0.023646 & 1.00 & 0.020690 & 1.00 & 0.017734 & 1.00 \\
\hline $1 \mathrm{~A}_{2}$ & 9.16 & 0.0004 & 0.000391 & 0.000292 & 9.15 & 0.000180 & 0.37 & 0.000157 & 0.40 & 0.000135 & 0.46 \\
\hline $2 \mathrm{~A}_{1}$ & 9.74 & 0.007563 & 0.011340 & 0.015116 & 9.74 & 0.007645 & 1.01 & 0.011462 & 1.01 & 0.015280 & 1.01 \\
\hline $2 \mathrm{~B}_{2}$ & 10.01 & 0.000759 & 0.000576 & 0.000393 & 10.01 & 0.000053 & 0.07 & 0.000047 & 0.08 & 0.000040 & 0.10 \\
\hline $3 \mathrm{~A}_{1}$ & 10.10 & 0.001288 & 0.001930 & 0.002573 & 10.10 & 0.001314 & 1.02 & 0.001970 & 1.02 & 0.002626 & 1.02 \\
\hline $3 \mathrm{~B}_{2}$ & 10.39 & 0.000253 & 0.000222 & 0.000190 & 10.38 & 0.000257 & 1.02 & 0.000225 & 1.01 & 0.000193 & 1.01 \\
\hline $2 \mathrm{~A}_{2}$ & 10.80 & 0.000015 & 0.000015 & 0.000015 & 10.79 & 0.000029 & 1.90 & 0.000025 & 1.70 & 0.000021 & 1.47 \\
\hline $4 \mathrm{~B}_{2}$ & 11.21 & 0.000159 & 0.000120 & 0.000082 & 11.21 & 0.000009 & 0.05 & 0.000007 & 0.06 & 0.000006 & 0.08 \\
\hline $4 \mathrm{~A}_{1}$ & 11.22 & 0.000011 & 0.000015 & 0.000020 & 11.21 & 0.000011 & 0.99 & 0.000016 & 1.04 & 0.000020 & 1.04 \\
\hline $5 \mathrm{~B}_{2}$ & 11.30 & 0.000062 & 0.000054 & 0.000046 & 11.30 & 0.000060 & 0.96 & 0.000052 & 0.97 & 0.000045 & 0.98 \\
\hline $3 \mathrm{~A}_{2}$ & 11.43 & 0.0000 & 0.000030 & 0.000021 & 11.42 & 0.000010 & 0.25 & 0.000008 & 0.28 & 0.000007 & 0.34 \\
\hline $1 \mathrm{~B}_{1}$ & 11.50 & 0.000430 & 0.000339 & 0.000248 & 11.50 & 0.000130 & 0.30 & 0.000114 & 0.34 & 0.000097 & 0.39 \\
\hline $6 \mathrm{~B}_{2}$ & 11.68 & 0.000092 & 0.000072 & 0.000051 & 11.67 & 0.000016 & 0.18 & 0.000014 & 0.20 & 0.000012 & 0.24 \\
\hline $5 \mathrm{~A}_{1}$ & 11.69 & 0.000002 & 0.000003 & 0.000004 & 11.69 & 0.000002 & 1.21 & 0.000003 & 1.10 & 0.000004 & 1.10 \\
\hline $7 \mathrm{~B}_{2}$ & 11.72 & 0.000076 & 0.000060 & 0.000043 & 11.71 & 0.000016 & 0.21 & 0.000014 & 0.23 & 0.000012 & 0.27 \\
\hline $4 \mathrm{~A}_{2}$ & 11.83 & 0.00015 & 0.000117 & 0.000079 & 11.81 & 0.000005 & 0.03 & 0.000004 & 0.04 & 0.000004 & 0.05 \\
\hline $8 \mathrm{~B}_{2}$ & 12.20 & 0.000641 & 0.000483 & 0.000324 & 12.20 & 0.000013 & 0.02 & 0.000012 & 0.02 & 0.000010 & 0.03 \\
\hline $6 \mathrm{~A}_{1}$ & 12.26 & 0.000046 & 0.000067 & 0.000088 & 12.26 & 0.000045 & 0.98 & 0.000066 & 0.98 & 0.000087 & 0.98 \\
\hline $7 \mathrm{~A}_{1}$ & 12.66 & 0.000113 & 0.000169 & 0.000224 & 12.66 & 0.000118 & 1.04 & 0.000175 & 1.04 & 0.000233 & 1.04 \\
\hline $9 \mathrm{~B}_{2}$ & 13.07 & 0.000298 & 0.000247 & 0.000195 & 13.06 & 0.000191 & 0.64 & 0.000167 & 0.68 & 0.000144 & 0.74 \\
\hline
\end{tabular}

quently, RIXS spectra for water can only be quantitatively understood when nuclear degrees 
of freedom are properly included in the simulation, as was done in Ref. 22 and 90.

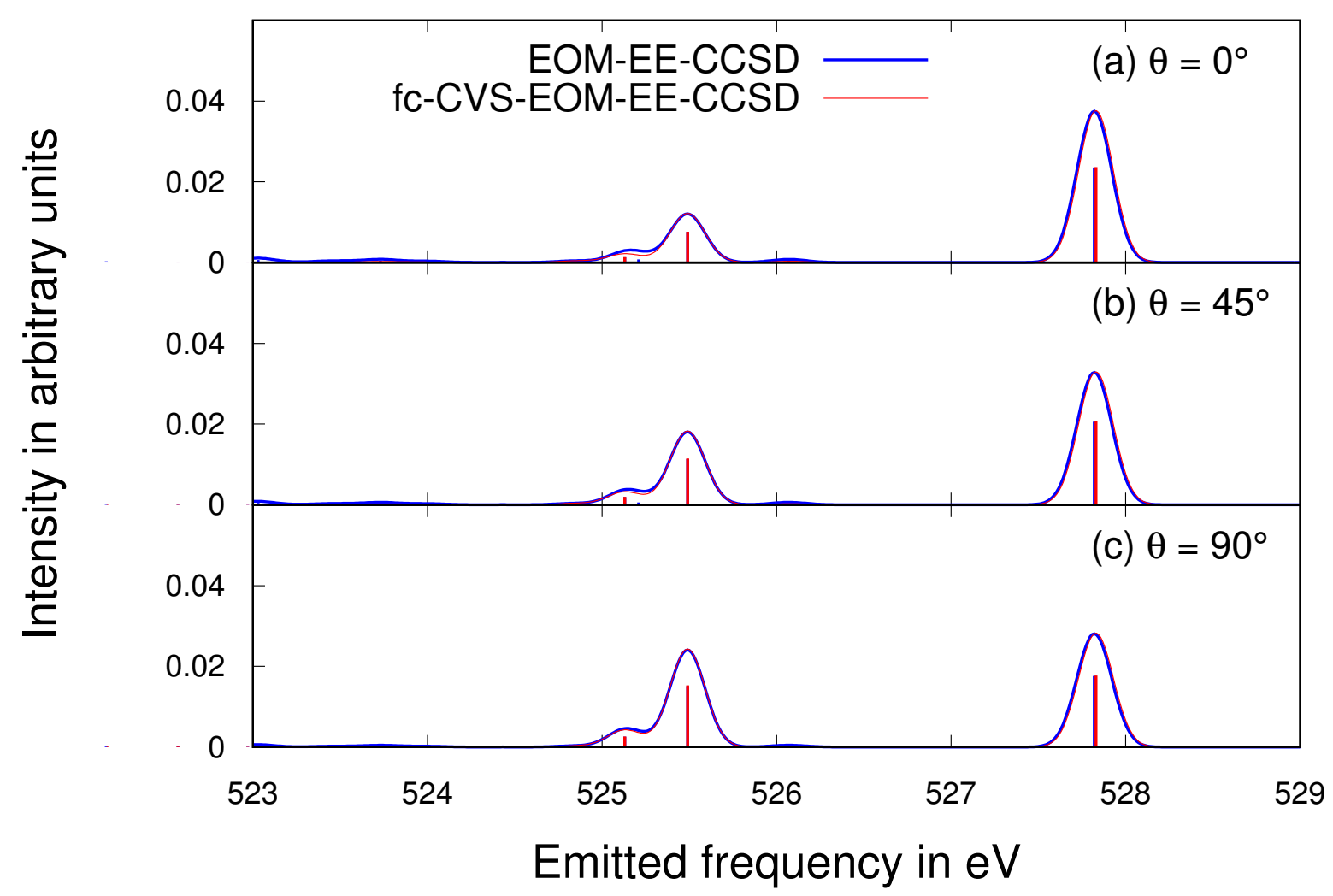

FIG. 3: Comparison of EOM-EE-CCSD and fc-CVS-EOM-EE-CCSD RIXS spectra for the incident photon resonant with the $1 s \rightarrow 4 a_{1}$ core excitation in water $(\mathrm{FWHM}=0.33 \mathrm{eV})$. (a) $\theta=0^{\circ}$, (b) $\theta=45^{\circ}$, (c) $\theta=90^{\circ}$

\section{B. Illustrative calculation: RIXS of benzene and benzene cation}

\section{Benzene}

Modeling X-ray spectra of benzene is challenging due to its high symmetry, multiple core orbitals, and the need for very diffuse functions to describe Rydberg excited states. Figure 4 shows occupied molecular orbitals of benzene; the respective vertical ionization energies (IEs) computed with (CVS)-EOM-IP-CCSD at the geometry of the neutral are collected in Table S2 of the SI. In this case, the correlated Dyson orbitals are visually indistinguishable from the canonical Hartree-Fock molecular orbitals and the correlated EOM-IP states can 
be mapped onto the latter. In the discussion below, we use molecular orbitals from Fig. 4 to refer to many-body EOM-IP states and vice versa.

Due to symmetry, all six $1 \mathrm{~s}_{C}$ core orbitals of benzene are delocalized, however, the splittings of the respective IEs are small (all six lie within $0.08 \mathrm{eV}$ ) because of their compactness. Table S2 also lists the IEs of the valence orbitals; the lowest IE of $9.2 \mathrm{eV}$ corresponds to the degenerate pair of $\pi$ orbitals, which give rise to the Jahn-Teller distortion. These values of IEs provide the maximum range of energy loss expected in XES and RIXS calculations from valence-excited states; a higher energy loss may result from valence resonances.

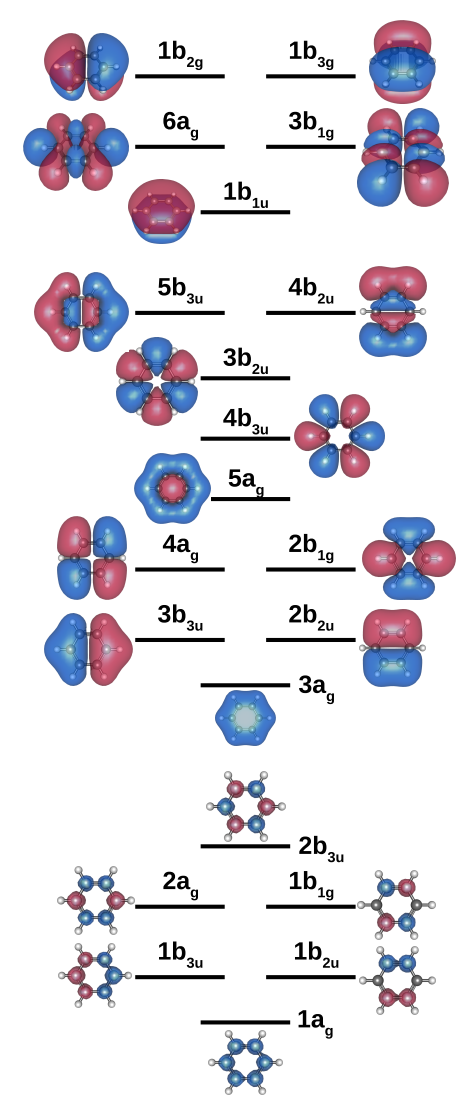

FIG. 4: Occupied molecular orbital diagram for benzene.

The XAS spectrum of neutral benzene is shown in Fig. 5; the respective transition energies and strengths are collected in Table S3 of the SI. The positions and relative intensities of the main peaks agree well with the experiment ${ }^{91,92}$ after a systematic shift of $-0.8 \mathrm{eV}$ is applied. Fig. 5 also shows the leading NTOs for the first two peaks; the NTOs for other transitions are collected in the SI. The NTOs reveal that peak A is derived from the $1 s_{C} \rightarrow \pi^{*}\left(\mathrm{~B}_{1 u}\right)$ transition, whereas doubly degenerate peak B corresponds to the 


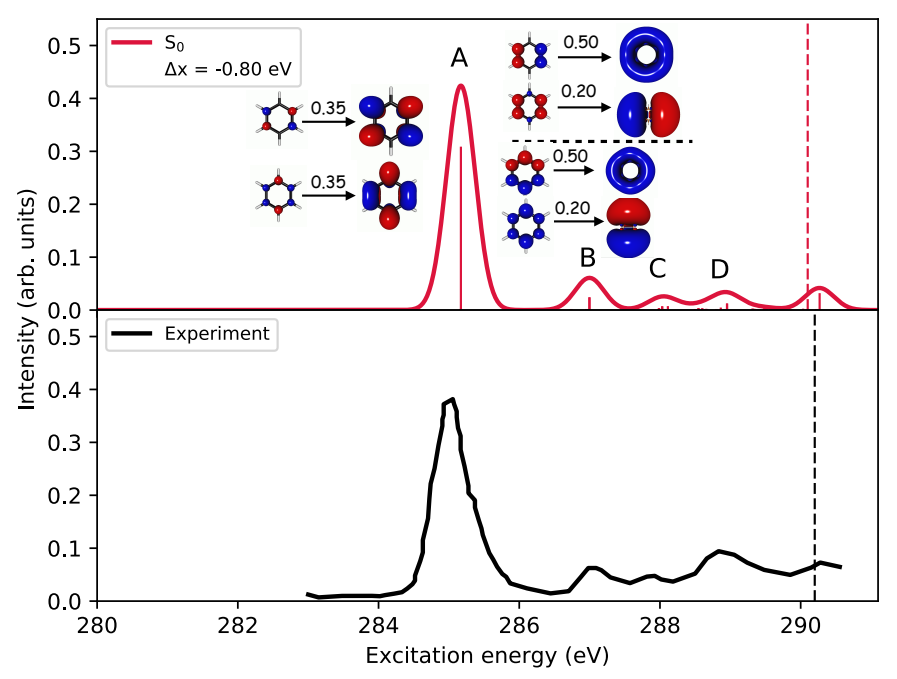

FIG. 5: XAS spectrum of benzene computed with fc-CVS-EOM-CCSD/6-311 $(2+,+) \mathrm{G}^{* *}(\mathrm{uC})$; FWHM $=0.8 \mathrm{eV}$. Dashed vertical lines correspond to the IEs. The energy shift required to align the NEXAFS profiles with the experimental one is $0.8 \mathrm{eV}$. The computed IE has been shifted by the same amount as used to align the NEXAFS profiles. NTOs and their weights are shown for peak A and peak B. The experimental spectrum is from Ref. ${ }^{91}$.

$1 s_{C} \rightarrow R y\left(\mathrm{~B}_{2 u} / \mathrm{B}_{3 u}\right)$ transition. The NTO analysis shows that excitation of peak A creates a hole in the $2 a_{g}$ and $1 b_{1 g}$ core orbitals, whereas the excitation of peak B creates holes in $1 b_{2 u} / 1 b_{3 u}$ and in an orbital, which can be described as a linear combination of $1 a_{g}$ and $2 a_{g}$. The weights of the NTOs (i.e., respective $\sigma^{2}$ values) quantify the relative contribution of each hole. Although the energies of the core orbitals giving rise to peak A and peak B are very close, their different symmetries have a profound effect on the shape of the RIXS emission spectra corresponding to pumping these two peaks.

The non-resonant X-ray emission of benzene could result from ionization of any of the six core orbitals. Thus, the XES spectrum can be computed as the sum of all possible transitions between the valence- and core-ionized states. The result is shown in Fig. 6. Apart from the overall shift of $-1.2 \mathrm{eV}$, the computed spectrum agrees very well with the experimental non-resonant emission spectrum ${ }^{93}$. As pointed out in Ref. 93, the structure of the XES spectrum can only be explained in terms of transitions to the delocalized core orbitals of proper symmetries (the localized $1 s$ holes would yield an entirely different pattern); our calculations provide a numeric confirmation of this conclusion. 


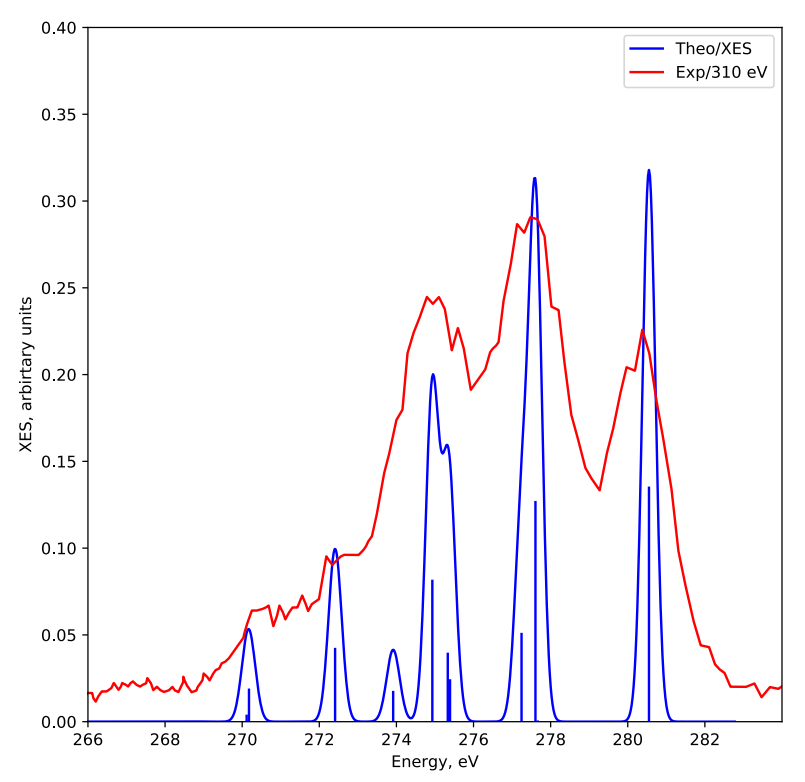

FIG. 6: XES transitions in the neutral benzene corresponding to ionization from all core orbitals. Blue: Theoretical spectrum computed with fc-CVS-EOM-IP-CCSD and fc-EOM-IP-CCSD and 6-311 $(2+,+) \mathrm{G}^{* *}(\mathrm{uC})$ and shifted by $1.2 \mathrm{eV}(\mathrm{FWHM}=0.4 \mathrm{eV})$. Red: experimental spectrum ${ }^{93}$ corresponding to $310 \mathrm{eV}$ excitation.

In contrast to XES, the RIXS emission spectrum is determined by the shape and symmetry of the specific core hole created in the excitation step as well as the (spectator) excited electron; we discuss these effects below. As suggested by Fig. 2, the RIXS process can be crudely described as a union of XAS excitation followed by the emission via XES-like transitions. For a specific core hole created in the excitation step (say, core hole A created by pumping peak A), assuming that the removal of the excited electron does not strongly perturb the $(N-1)$-electron core, the RIXS emision spectra of the neutral benzene can be approximated by computing the XES transitions between the specific core- and and all valence-ionized states. In other words, we synthesize an approximate RIXS emission spectrum by ignoring the effect of the spectator electron and by choosing the specific core hole that modulates the intensities of the XES peaks from Fig. 6. Below, we refer to the so-computed emission spectra as "poor man's RIXS emission". The purpose of these calculations is pedagogical: they illustrate the origin of the dependence of the RIXS emission spectra on the core hole (or pumping frequency) and provide simple molecular orbital picture of RIXS. Of course, we anticipate that not all features of the proper RIXS spectra will be 


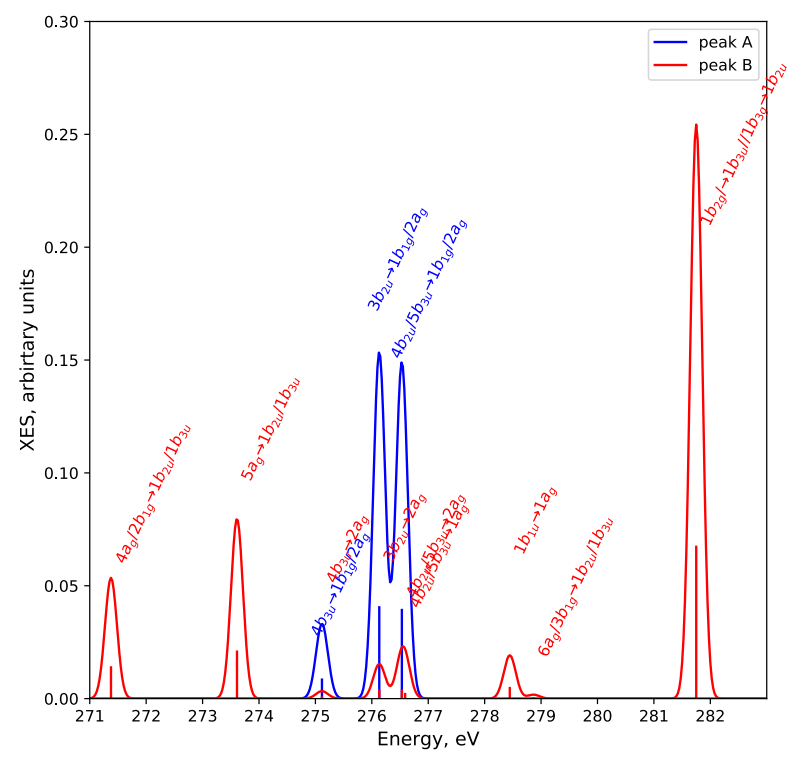

FIG. 7: Poor man's RIXS emission spectra of core-ionized benzene with the core holes of XAS peaks A (blue) and B (red) with $\mathrm{FWHM}=0.25 \mathrm{eV}$. The oscillator strengths are computed between fc-CVS-EOM-IP-CCSD and fc-EOM-IP-CCSD states with $6-311(2+,+) \mathrm{G}^{* *}(\mathrm{uC})$ basis set. Note that the actual computed XES spectra of benzene in Fig. 6 has the same peak positions (before the overall shift of $-1.2 \mathrm{eV}$ ) as in poor man's RIXS emission spectra but the intensities are different.

captured by such calculations; peak intensities and positions may be affected by the spectator electron. The comparison of the properly computed RIXS spectra with poor man's RIXS will highlight fundamental aspects of the RIXS process: its coherent two-photon nature and the effect of the spectator electron.

The NTO analysis of the XAS spectrum of benzene (Fig. 5 and Table S4 in the SI) shows the core hole orbitals corresponding to peaks A and B. Although the shapes of NTOs are slightly different from the Hartree-Fock orbitals (for example, the hole orbitals from the second NTO pairs of the two degenerate transitions giving rise to peak B show mixing of $1 a_{g}$ and $2 a_{g}$ canonical molecular orbitals from Fig. 4), one can still map the NTOs into the canonical and/or Dyson orbitals from Fig. 4. We use these orbitals (and the respective NTO weights) to compute the corresponding poor man's RIXS emission; the resulting emission spectra are shown in Fig. 7. The NTO analysis of the corresponding transitions is given in Table S5 of the SI; it confirms that the the molecular orbitals from Fig. 4 provide qualitatively correct description of the transitions. The two poor man's RIXS emission spectra show significant differences: the relaxation to core hole A yields only three features, 
whereas the relaxation to core hole B shows more features dominated by the one due to the relaxation of the $\mathrm{HOMO}$ to the core hole $\mathrm{B}$ around $282 \mathrm{eV}$. This is not surprising as the symmetry of the core hole dictates the symmetry of the valence orbitals that are active in poor man's RIXS (and proper RIXS) emission. For example, the presence of the $b_{3 u}$ core hole allows only the relaxation from the $a_{g}, b_{1 g}$, and $b_{2 g}$ valence levels (in $D_{2 h}$ group, only $\mathrm{B}_{1 u}, \mathrm{~B}_{2 u}$, and $\mathrm{B}_{3 u}$ transitions are optically allowed). These calculations suggest that the properly computed RIXS emission spectra should also show strong dependence on the pumping frequency.

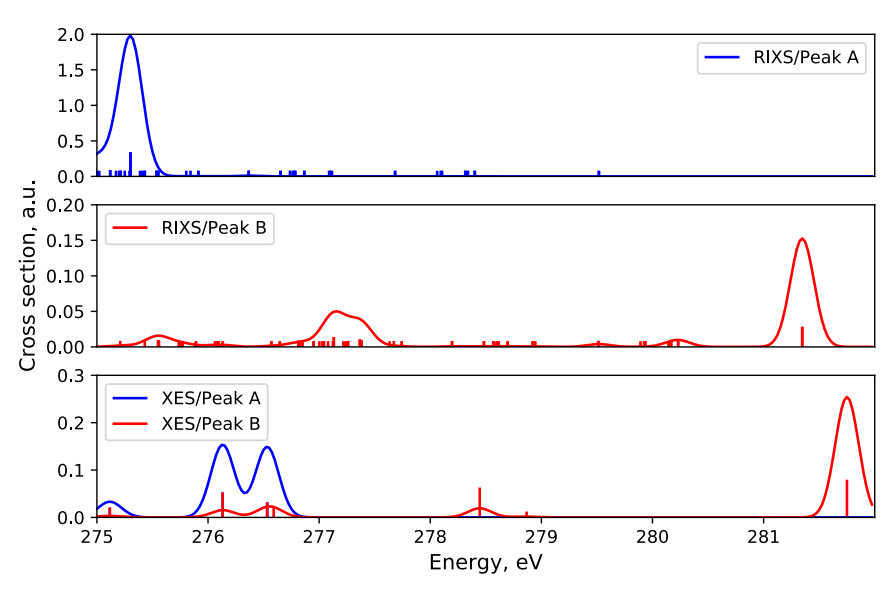

FIG. 8: Benzene: Computed RIXS spectra corresponding to pumping peak A (285.97 eV) and peak B $(287.80 \mathrm{eV})$ with $\theta=0$ and the corresponding poor man's RIXS emission spectra (FWHM=0.25 $\mathrm{eV}$ ). The positions of the excited states included in RIXS calculations are shown by sticks (sticks that give zero intensity are given some small finite height).

The properly computed RIXS emission spectra corresponding to pumping peak A and peak B computed with fc-CVS-EOM-EE-CCSD are shown in Fig. 8. The selection rules for the two-photon RIXS transitions in benzene $\left(D_{2 h}\right)$ allow only gerade transitions within the dipole approximation. The RIXS spectrum for the two pumping frequencies is computed using the same set of valence excited states (20 excited states per symmetry irreducible representation); their positions and contributions are shown by sticks in the top two panels of Fig. 8. Note that the positions of the sticks in the two panels are offset by $1.83 \mathrm{eV}$ by virtue of Eq. (1). The difference between the two RIXS spectra, corresponding to the resonant $1 s_{C} \rightarrow \pi^{*}$ core excitation (peak A) and $1 s_{C} \rightarrow R y$ core excitation (peak B), is stark. As one can see, the relative intensities of the RIXS lines corresponding to the same excited 
states are vastly different: compare, for example, the first line from the right corresponding to the doubly degenerate RIXS transitions to the $1 \mathrm{~B}_{2 g}$ and $1 \mathrm{~B}_{3 g}$ excited states at $6.45 \mathrm{eV}$ (HOMO-LUMO $\pi \rightarrow \pi^{*}$ excitation). Because of the different symmetry of the core hole created by pumping peak $\mathrm{A}$ and peak $\mathrm{B}$, these transitions have nearly zero intensity when peak $\mathrm{A}$ is pumped and become a dominant feature of the spectrum when peak B is pumped. This RIXS peak at $281.35 \mathrm{eV}$ emission energy for pumping peak B also appears in the corresponding poor man's RIXS emission spectrum at $281.75 \mathrm{eV}$, illustrating a spectator shift of $-0.40 \mathrm{eV}$. Similarly, the dominant RIXS transition for pumping peak A shows up at $275.30 \mathrm{eV}$ emission energy (10.67 eV energy loss) and corresponds to doubly degenerate transitions to $13 \mathrm{~B}_{2 g}$ and $12 \mathrm{~B}_{3 g}$ states. These excited-state RIXS transitions are the secondmost intense for the pumping frequency B and show up at $277.13 \mathrm{eV}$ emission energy. The poor man's RIXS emission spectra reproduces this feature, albeit at $276.53 \mathrm{eV}$. Thus, the spectator shifts for pumping frequencies $\mathrm{A}$ and $\mathrm{B}$ are $-1.23 \mathrm{eV}$ and $0.60 \mathrm{eV}$, respectively. The poor man's RIXS emission spectra for pumping frequency A shows a dominant feature at $276.13 \mathrm{eV}$ (labelled $3 b_{2 u} \rightarrow 1 b_{1 g} / 2 a_{g}$ ). In the properly computed RIXS spectrum, this feature due to the net transitions to the $16 \mathrm{~B}_{2 g}$ and $16 \mathrm{~B}_{3 g}$ states at $10.85 \mathrm{eV}$ energy loss is no longer as dominant; the primary reason being the smaller dipole coupling of the response state with the final state due to the presence of the spectator electron.

Fig. 9 shows the (properly computed) RIXS spectra corresponding to pumping peaks AD; the full two-dimensional RIXS energy-loss maps are shown in Fig. 10 and in Fig. S2 in the SI. The computed RIXS cross sections at each pumping frequencies are given in Tables S6, S7, and S8 of the SI. As expected, the brightest RIXS signal is obtained by pumping peak A, which dominates the XAS spectrum. Scanning the pumping frequency leads to pronounced changes in peak positions and relative intensities, as anticipated above. The RIXS spectrum obtained for pumping frequency A, in particular, shows stark differences compared to the spectra for higher energy pumping frequencies. For example, the RIXS peak around the $6.45 \mathrm{eV}$ energy loss does not appear for pumping frequency A. Also, the elastic scattering peak (at zero energy loss) and the inelastic peak at $10.67 \mathrm{eV}\left(1 \mathrm{~A}_{g} \rightarrow 13 \mathrm{~B}_{2 g} / 12 \mathrm{~B}_{3 g}\right)$ are the most intense at this incident frequency and are 1 to 2 orders of magnitude more intense than the brightest emissions for higher energy pumping frequencies, in agreement with the experimental findings ${ }^{93}$. The spectra corresponding to pumping peaks B, C1, C2, C3, and $\mathrm{D}$ appear very similar. The energy-loss peaks at $6.45 \mathrm{eV}\left(1 \mathrm{~A}_{g} \rightarrow 1 \mathrm{~B}_{2 g} / 1 \mathrm{~B}_{3 g}\right)$ and 10.67 


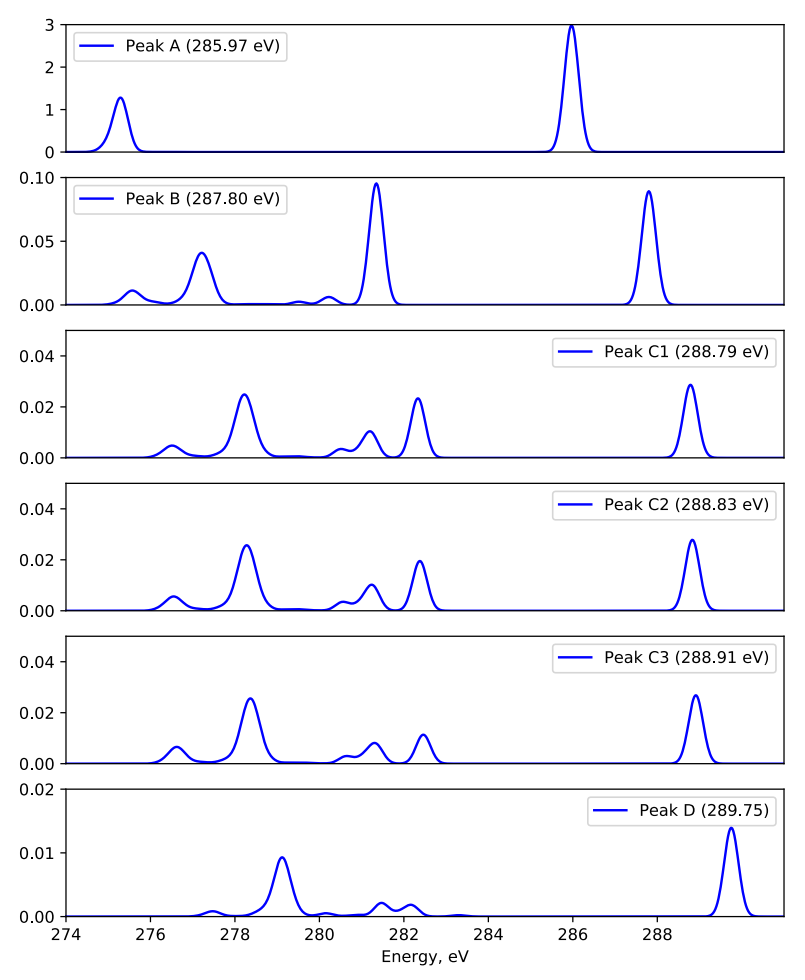

FIG. 9: RIXS spectra corresponding to excitation of peaks A-D computed with fc-CVS-EOMCCSD $/ 6-311(2+,+) \mathrm{G}^{* *}(\mathrm{uC}) ; \mathrm{FWHM}=0.4 \mathrm{eV}$. The $y$-axes shows absolute RIXS cross sections in atomic units.

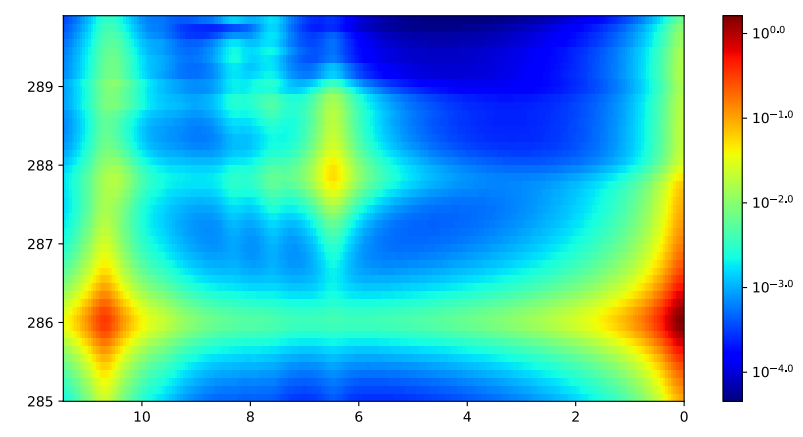

FIG. 10: Computed RIXS/REXS two-dimensional energy-loss ( $X$ axis) spectra with the intensities shown on the logarithmic scale; fc-CVS-EOM-CCSD/6-311 $(2+,+) \mathrm{G}^{* *}(\mathrm{uC})$.

$\mathrm{eV}\left(1 \mathrm{~A}_{g} \rightarrow 13 \mathrm{~B}_{2 g} / 12 \mathrm{~B}_{3 g}\right)$ are important in the RIXS spectra for pumping frequencies $\mathrm{B}$ and $\mathrm{C} 1 / \mathrm{C} 2 / \mathrm{C} 3$. The energy-loss peak at $6.45 \mathrm{eV}$, however, is not significant for pumping frequency D. For the $\mathrm{B}$ and $\mathrm{C} 1 / \mathrm{C} 2 / \mathrm{C} 3$ pumping frequencies, the energy-loss peak at 7.57 $\mathrm{eV}\left(1 \mathrm{~A}_{g} \rightarrow 11 \mathrm{~B}_{2 g} / 11 \mathrm{~B}_{3 g}\right)$ is also noticeable. 

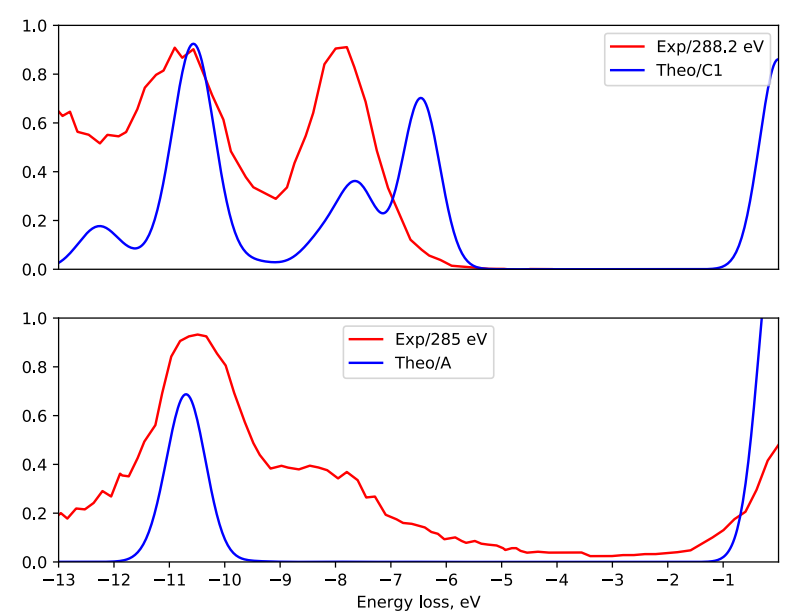

FIG. 11: Benzene RIXS energy-loss spectra (arbitrary intensities). Theory (blue): energy loss corresponding to pumping peaks $\mathrm{A}$ and $\mathrm{C} 1(\mathrm{FWHM}=0.8 \mathrm{eV})$. The experimental spectra ${ }^{93}$ are shown in blue.

We conclude this section by comparing the computed RIXS spectra with the available experimental data ${ }^{93-95}$. Fig. S3 in the SI shows the RIXS spectra from the gas-phase ${ }^{93}$ and condensed-phase studies ${ }^{94}$. As one can see, the spectra show significant changes as a function of pumping frequency. We also note that, despite general agreement between the two experiments, there are some discrepancies, especially in relative peak intensities. A close inspection of the available data shows that the scan at $285 \mathrm{eV}$ should correspond to pumping peak A and the other scans correspond to pumping peaks C-D. Unfortunately, the reported data ${ }^{93,94}$ do not include excitation frequency corresponding to peak $\mathrm{B}$. Thus, we limit our comparisons to the two sets from Ref. 93. Fig. 11 compares the computed RIXS spectra corresponding to pumping peaks A and C1 with the experimental energy loss spectra for 285 and $288.8 \mathrm{eV}$. As one can see, the agreement between theory and experiment is reasonably good - the positions of major features are reproduced well. In the low-energy spectrum, theory does not capture the shoulder at $-8.5 \mathrm{eV}$, whereas for the spectra at 288.2 $\mathrm{eV}$ pump, there is a discrepancy for the peak at $-8.0 \mathrm{eV}$. One possible explanation of these (relatively small) discrepancies is small mismatch between the reported experimental pumping frequencies and the XAS peak positions; given the strong dependence of the emission spectra on the pumping frequency, this can explain the disagreement. Alternatively, these difference may be due to nuclear motions, which are not included in the present theoretical framework and are believed to be sufficiently important in RIXS spectra of benzene ${ }^{94}$. 


\section{Benzene cation}

XAS and RIXS spectra of open-shell species derived by the ionization of closed-shell molecules acquire new, distinguishing features due to the presence of the valence hole. The excitation of the core electrons can populate this valence hole, giving rise to a characteristic lower energy peak in the XAS spectra of the open-shell species. The resonant excitation of this peak in RIXS provides a means to obtain the energy-loss spectrum characteristic of the open-shell species, which can be thus probed in the presence of the respective neutral parent molecules, even when the latter are present in great excess. In particular, the characteristic features of open-shell RIXS energy-loss spectra corresponding to excitation to the valence singly occupied orbital and the XES-like spectrum of the parent closed-shell molecule with the same core hole (poor man's RIXS for the cation) are expected to be similar, if computed at the same geometry. Note that this similarity between XES-like spectrum of the neutral and RIXS of the cation only holds for the excitation frequency that corresponds to the transition filling the valence hole; the RIXS emission spectra corresponding to excitations into higher lying unoccupied orbitals would be more similar to the emission spectra of the dication. Here, we illustrate these essential relationships between the closed-shell molecules and the respective ionized species by considering XAS and RIXS of benzene and its cation.

Benzene cation is a classic Jahn-Teller system ${ }^{83}$ with doubly degenerate (at the FranckCondon) geometry ground state. Jahn-Teller distortions (of $0.2 \mathrm{eV}$ ) lead to two nearly degenerate minima, with the barrier between them well below zero point energy ${ }^{83}$.

Fig. 12 shows the XAS spectra of the cation (computed for the ${ }^{2} \mathrm{~B}_{3 g}$ state) and the respective NTOs for the lowest spectral feature. At the geometry of the neutral, peak A corresponds to the excitation to the valence hole (transitions between $1 b_{2 u} / 1 b_{3 u}$ core and $1 b_{3 g} / 1 b_{2 g} \mathrm{HOMO}$ ), while peaks $\mathrm{C}$ and $\mathrm{D}$ resemble the transitions in the neutral species (i.e., peaks A and B of the XAS of the neutral). Structural relaxation has a rather small effect on the spectral shape: peak $\mathrm{A}$ is blue-shifted by $0.3 \mathrm{eV}$. The results for all spectral lines are given in Table S9 and S10 of the SI.

Fig. 13 compares the RIXS emission spectrum of the benzene cation computed for pumping transition corresponding to the excitation into the valence hole (i.e., with incident photon resonant with peak A of the cation) with the corresponding poor man's RIXS for the cation (i.e., the XES-like spectrum of benzene with the core hole in $1 b_{2 u} / 1 b_{3 u}$ orbitals). The RIXS 


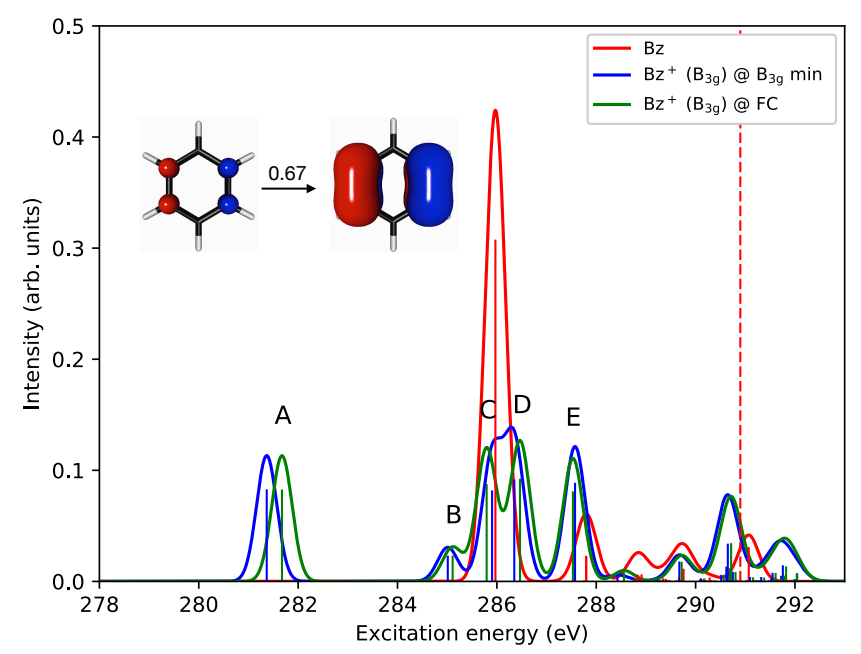

FIG. 12: XAS spectra of neutral benzene and its cation computed with fc-CVS-EOMEE-CCSD/6$311(2+,+) \mathrm{G}^{* *}(\mathrm{uC})$ at the Franck-Condon geometry and at the optimized geometry of the ${ }^{2} \mathrm{~B}_{3 g}$ ionized state; FWHM $=0.8 \mathrm{eV}$.

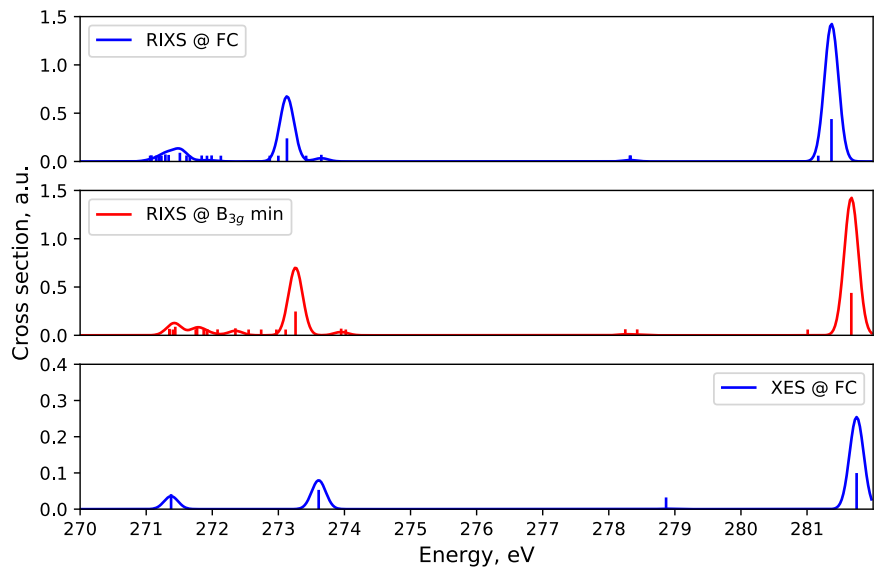

FIG. 13: Benzene cation $\left({ }^{2} \mathrm{~B}_{3 g}\right)$. RIXS spectra corresponding to the valence pump computed at the FC geometry (281.37 eV pump) and at the optimized geometry of the cation $\left({ }^{2} \mathrm{~B}_{3 g}\right.$ minimum, $281.67 \mathrm{eV}$ pump) at $\theta=0$ and the corresponding poor man's RIXS spectrum (marked as 'XES'). $\mathrm{FWHM}=0.25 \mathrm{eV}$.

spectra were computed by including the lowest 22 excited states below $10.35 \mathrm{eV}$. As expected, the two dominant features are similar - the elastic peak and the transition to the $2^{2} \mathrm{~A}_{g}$ state are slightly blue shifted by about $0.3 \mathrm{eV}$. Geometric relaxation has relatively small effect on the position and intensities of these two major features. The computed RIXS cross sections for benzene cation are presented in Table S11 of the SI. 


\section{CONCLUSIONS}

In this contribution, we present an extension of damped response theory for calculating RIXS cross sections using CVS within the response equations. Our primary aim was to address problematic divergence and erratic behavior of the response solutions in the X-ray frequency range, which is needed for RIXS calculations. This problematic behavior arises due to the coupling between virtual states to the decay channels into the valence-ionization continuum. We have extended a newly developed fc-CVS-EOM-EE-CCSD variant of the EOM-EE-CCSD theory to RIXS calculations by combining the damped response theory and the CVS truncation of the response space. This method exploits the ability of CVS to decouple the valence-excitation and core-excitation configurations of the excitation manifold, thereby blocking the autoionization decay channels for the virtual (i.e., response) states. The numeric results demonstrate that this approach solves the problematic divergence of the response solutions, while not affecting the quality of the computed RIXS spectrum. Our fcCVS-EOM-EE-CCSD implementation can treat both closed- and open-shell species, which we illustrated by calculating the RIXS emission spectra of (closed-shell) benzene and its (open-shell) cation. We analyzed the XAS, XES, and RIXS spectra of these species in terms of molecular orbitals and demonstrated the qualitative differences and similarities between their spectra. For neutral benzene, the computed spectra can be compared to the experimental ones, illustrating the robust performance of the fc-CVS-EOM-EE-CCSD approach. By providing an accurate and robust tool for computing electronic RIXS transition moments, fc-CVS-EOM-EE-CCSD serves as an excellent platform for further theoretical developments, such as the inclusion of nuclear motions, which is needed for a complete description of these phenomena.

\section{Supplementary Information}

Electronic Supplementary Information (ESI) available: programmable equations for iterative solutions of damped response equations, $\mathrm{LiH}$ data for validation of code with numerical sum-over-state RIXS moments, relevant Cartesian coordinates, raw data for XAS, XES, and RIXS spectra including relevant NTOs, basis set used for water, comparison of the experimental results for benzene. 


\section{Acknowledgments}

This work was supported by the U.S. National Science Foundation (No. CHE-1856342). A.I.K. is a grateful recipient of the Simons Fellowship in Theoretical Physics and Mildred Dresselhaus Award from CFEL/DESY, which supported her sabbatical stay in Germany. M.L.V., R.F., and S.C. acknowledge financial support from DTU Chemistry and from the Independent Research Fund Denmark-Natural Sciences, DFF-RP2 grant no. 7014-00258B. S.C. also acknowledges the European Union's Horizon 2020 Research and Innovation Programme under the Marie Sklodowska-Curie Grant Agreement No. 765739, "COSINE- European Training Network on COmputational Spectroscopy In Natural sciences and Engineering."

1 F. Gel'mukhanov and H. A. rgen, Phys. Rep., 1999, 312, 87-330.

2 S. Butorin, J. Electron. Spectrosc. Relat. Phenom., 2000, 110-111, 213-233.

3 F. de Groot, Chem. Rev., 2001, 101, 1779-1808.

4 A. Kotani and S. Shin, Rev. Mod. Phys., 2001, 73, 203-246.

${ }^{5}$ L. Ament, M. van Veenendaal, T. P. Devereaux, J. Hill and J. van den Brink, Rev. Mod. Phys., 2011, 83, 705-767.

6 T. Schmitt, F. de Groot and J. Rubensson, J. Synchrotron Rad., 2014, 21, 1065-1076.

7 S. Eckert, J. Norell, P. Miedema, M. Beye, M. Fondell, W. Quevedo, B. Kennedy, M. Hantschmann, A. Pietzsch, B. VanKuiken, M. Ross, M. Minitti, S. Moeller, W. Schlotter, M. Khalil, M. Odelius and A. Föhlisch, Phys. Rev. Lett., 2017, 56, 6088-6092.

8 Synchrotron Radiation: Basics, Methods and Applications, ed. S. Mobilio, F. Boscherini and C. Meneghini, Springer, 2014.

9 X-Ray Absorption and X-ray Emission Spectroscopy; Theory and Applications, ed. J. van Bokhoven and C. Lamberti, Wiley \& Sons, 2016.

10 X-Ray Free Electron Lasers: Applications in Materials, Chemistry and Biology, ed. U. Bergmann, V. Yachandra and J. Yano, Royal Society of Chemistry, 2017.

11 M. Nisoli, P. Decleva, F. Calegari, A. Palacios and F. Martín, Chem. Rev., 2017, 117, 1076010825. 
12 P. Norman and A. Dreuw, Chem. Rev., 2018, 118, 7208-7248.

13 T. Fransson, Y. Harada, N. Kosugi, N. Besley, B. Winter, J. Rehr, L. Pettersson and A. Nilsson, Chem. Rev., 2016, 116, 7551-7569.

14 O. Kostko, B. Bandyopadhyay and M. Ahmed, Annu. Rev. Phys. Chem., 2016, 67, 19-40.

15 D. Zuev, E. Vecharynski, C. Yang, N. Orms and A. I. Krylov, J. Comput. Chem., 2015, 36, $273-284$.

16 S. Coriani, O. Christiansen, T. Fransson and P. Norman, Phys. Rev. A, 2012, 85, 022507.

17 S. Coriani, T. Fransson, O. Christiansen and P. Norman, J. Chem. Theory Comput., 2012, 8, 1616-1628.

18 A. Sadybekov and A. I. Krylov, J. Chem. Phys., 2017, 147, 014107.

19 M. L. Vidal, X. Feng, E. Epifanovski, A. I. Krylov and S. Coriani, J. Chem. Theory Comput., 2019, 15, 3117-3133.

20 M. Lundberg, T. Kroll, S. DeBeer, U. Bergmann, S. A. Wilson, P. Glatzel, D. Nordlund, B. Hedman, K. O. Hodgson and E. I. Solomon, J. Am. Chem. Soc., 2013, 135, 17121-17134.

21 D. Maganas, P. Kristiansen, L.-C. Duda, A. Knop-Gericke, S. DeBeer, R. Schlögl and F. Neese, J. Phys. Chem. C, 2014, 118, 20163-20175.

22 E. Ertan, V. Savchenko, N. Ignatova, V. Vaz da Cruz, R. C. Couto, S. Eckert, M. Fondell, M. Dantz, B. Kennedy, T. Schmitt, A. Pietzsch, A. Föhlisch, F. Gel'mukhanov, M. Odelius and V. Kimberg, Phys. Chem. Chem. Phys., 2018, 20, 14384-14397.

23 D. Rehn, A. Dreuw and P. Norman, J. Chem. Theory Comput., 2017, 13, 5552-5559.

24 R. Faber and S. Coriani, J. Chem. Theory Comput., 2019, 15, 520-528.

25 A. Buckingham and P. Fischer, Phys. Rev. A, 2000, 61, 035801-035804.

26 K. Kristensen, J. Kauczor, A. J. Thorvaldsen, P. Jørgensen, T. Kjaergaard and A. Rizzo, J. Chem. Phys., 2011, 134, 214104-214120.

27 P. Norman, Phys. Chem. Chem. Phys., 2011, 12, 20519-20535.

28 J. Kauczor, P. Norman, O. Christiansen and S. Coriani, J. Chem. Phys., 2013, 139, 211102.

29 L. Jensen, J. Autschbach and G. C. Schatz, J. Chem. Phys., 2005, 122, 224115.

30 J. Kauczor and P. Norman, J. Chem. Theory Comput., 2014, 10, 2449-2455.

31 D. Maganas, S. DeBeer and F. Neese, Inorg. Chem., 2017, 56, 11819-11836.

32 N. Besley, A. Gilbert and P. Gill, J. Chem. Phys., 2009, 130, 124308.

33 N. A. Besley, Chem. Phys. Lett., 2012, 542, 42-46. 
34 K. Emrich, Nucl. Phys., 1981, A351, 379-396.

35 J. F. Stanton and R. J. Bartlett, J. Chem. Phys., 1993, 98, 7029-7039.

36 A. I. Krylov, Annu. Rev. Phys. Chem., 2008, 59, 433-462.

37 R. J. Bartlett, Mol. Phys., 2010, 108, 2905-2920.

38 K. Sneskov and O. Christiansen, WIREs: Comput. Mol. Sci., 2012, 2, 566-584.

39 R. J. Bartlett, WIREs: Comput. Mol. Sci., 2012, 2, 126-138.

40 J. Pople, Energy, Structure and Reactivity: Proceedings of the 1972 Boulder Summer Research Conference on Theoretical Chemistry, Wiley, New York, 1973, pp. 51-61.

41 L. Slipchenko, J. Phys. Chem. A, 2010, 114, 8824-8830.

42 O. Christiansen, J. Gauss and B. Schimmelpfennig, Phys. Chem. Chem. Phys., 2000, 2, 965-971.

43 K. Klein and J. Gauss, J. Chem. Phys., 2008, 129, 194106.

44 E. Epifanovsky, K. Klein, S. Stopkowicz, J. Gauss and A. I. Krylov, J. Chem. Phys., 2015, 143, 064102 .

45 P. Pokhilko, E. Epifanovsky and A. Krylov, J. Chem. Phys., 2019, 151, 034106.

46 A. Tajti and P. Szalay, J. Chem. Phys., 2009, 131, 124104.

47 T. Ichino, J. Gauss and J. F. Stanton, J. Chem. Phys., 2009, 130, 174105.

48 S. Faraji, S. Matsika and A. I. Krylov, J. Chem. Phys., 2018, 148, 044103.

49 T. Helgaker, S. Coriani, P. Jørgensen, K. Kristensen, J. Olsen and K. Ruud, Chem. Rev., 2012, 112, 543-631.

50 K. D. Nanda and A. I. Krylov, J. Chem. Phys., 2015, 142, 064118.

51 K. D. Nanda and A. I. Krylov, J. Chem. Phys., 2017, 146, 224103.

52 K. Nanda and A. I. Krylov, J. Phys. Chem. Lett., 2017, 8, 3256-3265.

53 K. Nanda and A. I. Krylov, J. Chem. Phys., 2018, 149, 164109.

54 M. de Wergifosse, C. G. Elles and A. I. Krylov, J. Chem. Phys., 2017, 146, 174102.

55 M. de Wergifosse, A. L. Houk, A. I. Krylov and C. G. Elles, J. Chem. Phys., 2017, 146, 144305.

56 P. B. Rozyczko, S. A. Perera, M. Nooijen and R. J. Bartlett, J. Chem. Phys., 1997, 107, $6736-6747$.

57 C. Hättig, O. Christiansen, S. Coriani and P. Jørgensen, J. Chem. Phys., 1998, 109, 9237.

58 K. D. Nanda and A. I. Krylov, J. Chem. Phys., 2016, 145, 204116.

59 K. Nanda, A. I. Krylov and J. Gauss, J. Chem. Phys., 2018, 149, 141101.

60 H. Koch and P. Jørgensen, J. Chem. Phys., 1990, 93, 3333-3344. 
61 T. B. Pedersen and H. Koch, J. Chem. Phys., 1997, 106, 8059-8072.

62 O. Christiansen, P. Jørgensen and C. Hättig, Int. J. Quant. Chem., 1998, 68, 1-52.

63 B. Peng, P. Lestrange, J. Goings, M. Caricato and X. Li, J. Chem. Theory Comput., 2015, 11, $4146-4153$.

64 S. Coriani and H. Koch, J. Chem. Phys., 2015, 143, 181103.

65 S. Coriani and H. Koch, J. Chem. Phys., 2016, 145, 149901.

66 H. Sekino and R. J. Bartlett, Int. J. Quant. Chem., 1984, 26, 255-265.

67 H. Koch, H. Jensen, P. Jørgensen and T. Helgaker, J. Chem. Phys., 1990, 93, 3345-3350.

68 L. Cederbaum, W. Domcke and J. Schirmer, Phys. Rev. A, 1980, 22, 206.

69 R. Myhre, S. Coriani and H. Koch, J. Chem. Theory Comput., 2016, 12, 2633-2643.

70 B. Tenorio, T. Moitra, M. Nascimento, A. Rocha and S. Coriani, J. Chem. Phys., 2019, 150, 224104 .

71 R. Faber and S. Coriani, Phys. Chem. Chem. Phys., 2019.

72 A. I. Krylov and P. M. W. Gill, WIREs: Comput. Mol. Sci., 2013, 3, 317-326.

73 Shao, Y.; Gan, Z.; Epifanovsky, E.; Gilbert, A.T.B.; Wormit, M.; Kussmann, J.; Lange, A.W.; Behn, A.; Deng, J.; Feng, X., et al., Mol. Phys., 2015, 113, 184-215.

74 F. Gel'mukhanov and H. Ågren, Phys. Rev. A, 1994, 49, 4378-4389.

75 C. Hättig, O. Christiansen and P. Jørgensen, J. Chem. Phys., 1998, 108, 8331-8354.

76 M. Paterson, O. Christiansen, F. Pawlowski, P. Jørgensen, C. Hättig, T. Helgaker and P. Salek, J. Chem. Phys., 2006, 124, 054332.

77 In the exact expression of the RIXS scattering moment, the damping factor in each sum-overstate term corresponds to the inverse lifetime of the intermediate state. In damped response theory, however, a phenomenological non-state-specific value for the damping factor is usually used.

78 P. Pulay, Chem. Phys. Lett., 1980, 73, 393.

79 G. Scuseria, T. Lee and H. Schaefer, Chem. Phys. Lett., 1986, 130, 236-239.

80 H. Feshbach, Ann. Phys. (N.Y.), 1962, 19, 287-313.

81 V. Averbukh and L. Cederbaum, J. Chem. Phys., 2005, 123, 204107.

82 H. Tachikawa, J. Phys. Chem. A, 2018, 122, 4121-4129.

83 R. Lindner, K. Müller-Dethlefs, E. Wedum, K. Haber and E. Grant, Science, 1996, 271, 16981702. 
84 R. Mulliken, J. Chem. Phys., 1955, 23, 1997-2011.

85 F. Plasser, M. Wormit and A. Dreuw, J. Chem. Phys., 2014, 141, 024106-13.

86 C. M. Oana and A. I. Krylov, J. Chem. Phys., 2007, 127, 234106-14.

87 M. L. Vidal, A. I. Krylov and S. Coriani, Phys. Chem. Chem. Phys., 2019.

88 E. Epifanovsky, M. Wormit, T. Kuś, A. Landau, D. Zuev, K. Khistyaev, P. U. Manohar, I. Kaliman, A. Dreuw and A. I. Krylov, J. Comput. Chem., 2013, 34, 2293-2309.

89 E. Kamarchik, O. Kostko, J. M. Bowman, M. Ahmed and A. I. Krylov, J. Chem. Phys., 2010, $132,194311$.

90 V. Vaz da Cruz, F. Gel'mukhanov, S. Eckert, M. Iannuzzi, E. Ertan, A. Pietzsch, R. C. Couto, J. Niskanen, M. Fondell, M. Dantz, T. Schmitt, X. Lu, D. McNally, R. M. Jay, V. Kimberg, A. Föhlisch and M. Odelius, Nat. Comm., 2019, 10, 1013.

91 D. Menzel, G. Rocker, H.-P. Steinrück, D. C. P. A. Heimann, W. Huber, P. Zebisch and D. R. Lloyd, J. Chem. Phys., 1992, 96, 1724.

92 E. E. Rennie, B. Kempgens, H. M. Köppe, U. Hergenhahn, J. Feldhaus, B. S. Itchkawitz, A. L. D. Kilcoyne, A. Kivimäki, K. Maier, M. N. Piancastelli, M. Polcik, A. Rüdel and A. M. Bradshaw, J. Chem. Phys., 2000, 113, 7362-7375.

93 P. Skytt, J. Guo, N. Wassdahl, J. Nordgren, Y. Luo and H. Ågren, Phys. Rev. A, 1995, 52, $3572-3576$.

94 F. Hennies, S. Polyutov, I. Minkov, A. Pietzsch, M. Nagasono, H. Ågren, L. Triguero, M.-N. Piancastelli, W. Wurth, F. Gelmukhanov and A. Föhlisch, Phys. Rev. A, 2007, 76, 032505.

95 J.-H. Guo, M. Magnuson, C. Såthe, J. Nordgren, L. Yang, Y. Luo, H. Ågren, K. Z. Xing, N. Johansson, W. R. Salaneck, R. Daik and W. J. Feast, J. Chem. Phys., 1998, 108, 59905996. 